\title{
Kinesiologic Tape Breaking the Traditional Pharmacologic Management of Postoperative Morbidity
}

\author{
${ }^{1}$ Abhishek Dutta, ${ }^{2}$ AL Gopinath, ${ }^{3}$ MA Reyazulla, ${ }^{4}$ Keerthi Ramesh, ${ }^{5}$ Shatakshi Srivastava, ${ }^{6}$ Shruthi Agarwal
}

\begin{abstract}
Objectives: Zygomatic maxillary complex fractures are common in trauma affecting maxillofacial region. Surgical treatment of these fractures causes pain, trismus and swelling, invariably affecting quality of life of patient postoperatively. To control these effects, various pharmacologic methods have been tried, but in vain. The kinesiologic tape (KT) opens the congested blood vessels and lymphatic channels, thereby improving flow across them. The goal of this study was to assess if the application of KT controls postoperative swelling as compared to corticosteroids.
\end{abstract}

Materials and methods: Twenty patients with zygomatic maxillary complex fractures, were randomly divided into two groups that is treatment either with $\mathrm{KT}$ or without $\mathrm{KT}$ using dexamethasone. Kinesiologic tape was placed starting immediately after surgery till 5 days postoperatively. Facial swelling was assessed at six specific time points, across by different lines on the face.

Results: Overall maximal swelling was seen among patients without the KT. Patients in the KT group showed maximal swelling in the immediate postoperative period, while patients in the no-KT group with dexamethasone had maximum swelling on day one. Also when we compare the extent of swelling on different days in the KT group, there is significant reduction in swelling $(p<0.05)$ compared to the other group.

Conclusion: Therefore, KT is a holistic, minimally invasive, economical approach which is improves patients' quality of life without the adverse effects of drugs.

Keywords: Kinesiologic tape, Trauma, Zygomatic complex.

How to cite this article: Dutta $A$, Gopinath $A L$, Reyazulla MA, Ramesh K, Srivastava S, Agarwal S. Kinesiologic Tape Breaking the Traditional Pharmacologic Management of Postoperative Morbidity. World J Dent 2015;6(4):199-202.

Source of support: Nil

Conflict of interest: None

\section{INTRODUCTION}

Midface fractures involving zygomatic complex region share a major workload in head and neck trauma. ${ }^{1-3}$ Surgical treatment of these fractures causes pain, trismus

\footnotetext{
${ }^{1,5,6}$ Postgraduate, ${ }^{2,3}$ Associate Professor, ${ }^{4}$ Professor

${ }^{1-6}$ Department of Oral and Maxillofacial Surgery, VS Dental College, Bengaluru, Karnataka, India
}

Corresponding Author: Abhishek Dutta, Postgraduate, Department of Oral and Maxillofacial Surgery, VS Dental College Bengaluru, Karnataka, India, Phone: 0802433890, e-mail: drabhishekmaxfac23@gmail.com and swelling, invariably affecting quality of life of patient postoperatively. ${ }^{4}$ Post surgery it is the tissue reaction that invariably causes the postoperative morbidity. ${ }^{5}$ The morbidity beomes a burden for the patient as it affects both the social and professional life and mandates the necessity to reduce these side effects, thus, improving patient's postoperative life. ${ }^{6}$ Several methods have been described in literature to control the inflammatory response associated with surgery, like the use of drugs, such as analgesics, 7,8 corticosteroids, laser application proteolytic enzymes or physical therapeutic methods like cryotherapy or manual lymph drainage (MLD). ${ }^{9}$ Due to their anti-inflammatory effects, glucocorticoids (GCs) are widely used to decrease tissue reactions that cause discomfort postoperatively. ${ }^{10}$ The interest in the use of GCs in association with oral and maxillofacial surgery was awakened in the 1950s. ${ }^{11}$

When GCs are used, even though the duration of medication is short, the doses are usually high. Potential complications of high-dose application of GCs are avascular necrosis, ${ }^{12}$ psychosis, ${ }^{13}$ gastrointestinal ulcer, and even gastrointestinal perforation and bleeding ${ }^{14}$ have been reported. In addition, GCs impair the immune defense by various mechanisms and short-term, highdose usage might have adverse effects, such as infections and impairment of surgical site healing. Therefore, further techniques for a better swelling control must be employed for patients who undergo zygomatic complex surgery.

Kinesiologic tape (KT) first introduced in 1970, and has become popular in the sport fraternity. The effects of Kinesiologic tape include giving support to muscles and joints, diminishing pain, and decreasing the collection of inflammatory fluid in the injured area. But at present, there is no evidence to support these effects and more research is necessary. ${ }^{15}$ The goal of this study is to assess if the application of KT prevents swelling as compared to the traditional method of using corticosteroids, thereby reducing the postoperative systemic and local complications associated with the use of corticosteroids.

\section{MATERIALS AND METHODS}

The protocol was reviewed by our institutional review board (IRB), is in compliance with the Helsinki declaration, and that each subject in the study signed a 
detailed informed consent form. The ethical clearance certificate was obtained from the ethical committee, before the commencement of the study.

\section{Patients}

A prospective, monocentric, parallel group, randomized clinical trial involving 20 male patients with age range (18-60 years) presenting with zygomatic complex fractures which required open reduction and internal fixation were randomized into two study groups with informed consent of the patient. All the patients had fracture involving the infraorbital rim, frontozygomatic suture and zygomatic buttress area which were laterally displaced with rotation along horizontal axis. The incisions used in all the patients were lateral eyebrow incision for frontozygomatic suture, subciliary incision for infraorbital rim and keens intraoral approach for zygomatic buttress area. Surgery in all the patients were done within 24 to 48 hours of injury.

\section{Group 1}

Patients in kinesiologic tape group.

\section{Group 2}

Patients in corticosteroid group.

\section{Inclusion Criteria}

Inclusion criteria were zygomatic maxillary complex fracture involving infraorbital margin, frontozygomatic suture and zygomatic buttress, uncomplicated ZMC fractures with minimal lateral displacement and rotation along horizontal axis.

\section{Exclusion Criteria}

Exclusion criteria were sensitivities to tape, cases managed conservatively, gross communition of orbital floor needing repair/reconstruction, zygomatic arch fracture.

\section{Operation}

All operations were performed by specialized oral and maxillofacial surgeons under general anesthesia following standardized operation protocol and using standardized approaches. Mean operation duration varied between 60 and 90 minutes.

\section{Group 1}

Patients in kinesiologic tape group.

The tape was applied by the same investigator in all the patients. Skin is cleaned of moisture and oils prior to application. a blue colored K-active tape classic was used in all the cases. Tape was applied for every patient

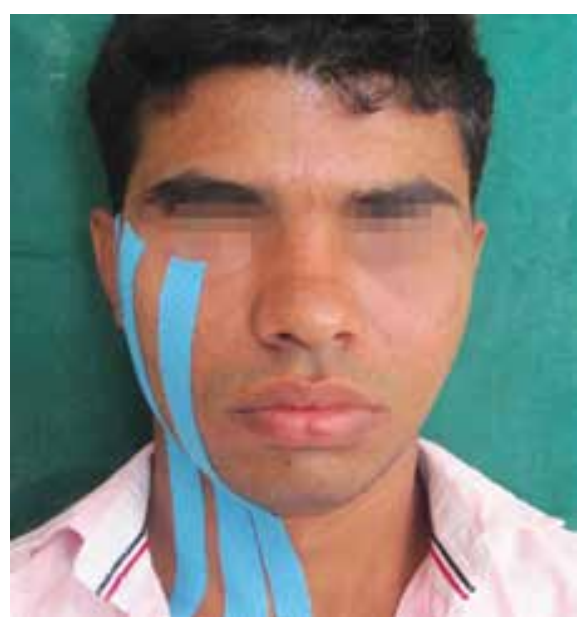

Fig. 1: Patient with kinesiologic tape

starting from clavicle to maximal swelling. The tape was divided in three equal strips before application. The skin was made taut, and the tapes were applied with slight stretch (20\%) (Fig. 1). The tape was kept for 5 days postoperatively.

\section{Group 2}

Patients in corticosteroid group.

Dexamethasone $8 \mathrm{mg}$ administered twice a day via intravenous route for 3 days followed by $4 \mathrm{mg}$ twice a day for the next 2 days.

\section{Measurements}

The swelling was assessed at different time intervals (T): immediately after operation (T0), and then every 24 hours postoperatively 1st day (T1), 2nd day (T2), 3rd day (T3), 4th day (T4), 5th day (T5).

\section{Swelling}

A standard plastic tape was used to measure the extent of swelling along five different lines as follows':

- Line a: Tragus to lip commissure

- Line b: Tragus to pogonion

- Line c: Tragus to lateral canthus of the eye

- Line d: Lateral canthus of the eye to gonion

- Line e: Gonion to nasion.

\section{RESULTS}

All swelling measurements are expressed in centimeters. The total swelling for each day was assessed by adding the values along five different lines described above. The statistical analysis is done using paired t-test. A bar diagram depicted in Graph 1 shows the maximal swelling plotted against time. The value of maximal swelling is greater in the non-KT group when compared to KT group as is evident from the bar diagram. Patients in the KT group showed maximal swelling immediate 


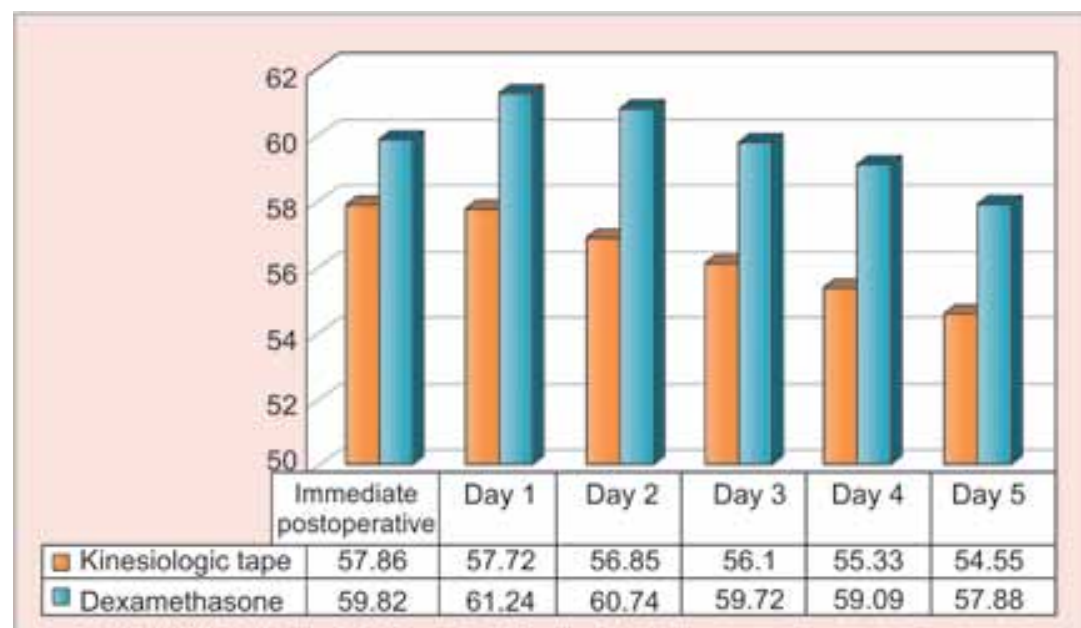

Graph 1: Maximal swelling on different days in the two groups

Table 1: Measurement of swelling on different days in the KT group

\begin{tabular}{llll}
\hline $\begin{array}{l}\text { Time of } \\
\text { assessment }\end{array}$ & $\begin{array}{l}\text { Mean } \pm \\
\text { standard } \\
\text { deviation }\end{array}$ & $\begin{array}{l}\text { Mean } \\
\text { difference }\end{array}$ & p-value \\
\hline $\begin{array}{l}\text { Immediate } \\
\text { postoperative }\end{array}$ & $57.86 \pm 5.28$ & & \\
Day 1 & $57.72 \pm 4.95$ & 0.06 & 0.496 \\
Day 2 & $56.85 \pm 4.79$ & 1.01 & 0.002 \\
Day 3 & $56.10 \pm 5.07$ & 1.76 & 0.000 \\
Day 4 & $55.33 \pm 4.92$ & 2.53 & 0.000 \\
Day 5 & $54.55 \pm 4.95$ & 3.31 & 0.000 \\
\hline
\end{tabular}

postoperatively while patients in the no-KT group with dexamethasone had maximum swelling on day one. Also when we compare the extent of swelling on different days in the KT group, there is significant reduction in swelling (Table 1).

\section{DISCUSSION}

Facial fractures that cause esthetic and/or functional disturbances have to be surgically reduced and fixed with plates and screws. The injury itself along with the needed surgical intervention causes swelling and pain, indicating the use of anti-inflammatory drugs during and after surgery. There are a plethora of methods available to control postoperative edema in maxillofacial surgery. Laser is a newer modality reducing postoperative discomfort, especially swelling after third molar surgery. ${ }^{16}$ Ice therapy is a simple and cheap modality. Its therapeutic effects are attributed to consequent vasoconstriction causing decreased blood flow and metabolism, thereby minimizing bacterial growth. But data proving the effect of cryotherapy on swelling are controversial. ${ }^{17}$

The interest in the use of steroids in association with oral and maxillofacial surgery was awakened in the $1950 .{ }^{11}$ The anti-inflammatory actions of steroids are well-known. Their effects are mediated by multiple mechanisms, and the network of the cascades is extensive. Suppression of inflammation is caused by a diminished secretion of proteolytic and lipolytic enzymes, reduced release of vasoactive and chemoattractive factors, decreased fibrosis, a lower number of circulating lymphocytes, and reduced extravasation of leucocytes to the site of injury. The association between steroids and disturbance in surgical site healing after oral surgery was discussed already in the late 1960s by Hooley et al (1969). Steroids alter the functions and concentrations of cells critical in inflammatory response and tissue repair. The altered cellular function caused by steroids is transmitted through induction of gene transcription. The steroid receptor interacts with proteins, which control proinflammatory gene expression, inducing diminished healing. Steroids can also induce apoptosis. In addition to direct cellular influences, there are several other steroid-related mechanisms that should be taken into consideration in surgical wound healing. ${ }^{18}$

Steroids affect the concentration of blood cells by decreasing the numbers of circulating lymphocytes, eosinophils, basophils, and monocytes and increasing the numbers of circulating polymorphonuclear granulocytes and stab cells. ${ }^{19}$ Glucocorticoids also increase blood glucose levels and inhibit the proliferation of fibroblasts. ${ }^{20}$ Moreover, GCs decrease collagen synthesis and levels of growth factors in wounds. ${ }^{21}$ Also dexamethasone may mask the signs of infection and inflammation, and therefore, clinical verification of local disturbances may occur with a lag. Short-term dexamethasone decreases inflammatory mediator release effectively. ${ }^{22}$

In a study done on 64 subjects having zygomatic complex fractures disturbance in surgical wound healing occurred significantly more often in patients who received steroids (24.2\%) than in those who did not (3.2\%) $(p=0.016){ }^{18}$ 
Due to the above explained evidence based side effects associated with steroids, management with better alternatives are always sought after by surgeons has been increasingly used in the rehabilitation protocols and prevention of sports injuries. The results of this study show that the application of KT after zygomatic complex surgery has a significant influence on reduction of swelling as compared to the pharmacologic method using dexamethasone. This might be attributed to KT elasticity, thickness and adhesion allowing it to mimic the quality of the skin. The KT has been designed to allow 30 to $40 \%$ increase in length when stretched. Tension is induced in the KT by stretching it and the tape subsequently applied to the skin. As the KT recoils back to its original length following the application, it creates a upward traction to the skin forming convolutions beneath the tape. It is thought that these convolutions relieve pressure on the congested blood vessels and lymphatic channels, thus increasing the flow of blood and lymphatic fluid. According to Shim et al who did a pilot study on animals these convolutions elevate the skin. They proposed that this convolutions lead to opening of micro valves in the interstitial lymphatic vessels, thereby generating adequate dynamic pressure variation and sufficient periodic compression and decompression of superficial and deep interstitial lymphatics. ${ }^{9}$ There are no reported cases of severe allergic reaction to KT application.

\section{CONCLUSION}

Therefore, KT is a holistic, minimally invasive, economical and can be performed anywhere in the world. It can be used as a substitute for corticosteroids which has many side effects in addition to increased cost of administration and prolonged hospital stay which eventually affects the social and economic aspects of the patient. Thus, KT can be used to break or substitute the traditional pharmacologic management of postoperative morbidity after zygomatic complex surgery.

\section{REFERENCES}

1. van den Bergh B, Karagozoglu KH, Heymans MW, Forouzanfar T. Aetiology and incidence of maxillofacial trauma in Amsterdam: a retrospective analysis of 579 patients. J Craniomaxillofac Surg 2012;40:165-169.

2. Rogers SN, Naylor R, Potter L, Magennis P. Three years' experience of collaborative care pathways on a maxillofacial ward. Br J Oral Maxillofac Surg 2000;38:132-137.

3. Naveen Shankar A, Naveen Shankar V, Hegde N, Sharma RP. The pattern of the maxillofacial fractures: a multicentre retrospective study. J Craniomaxillofac Surg 2012;40:675-679.

4. Trivellato PF, Arnez MF, Sverzut CE, Trivellato AE. A retrospective study of zygomatico-orbital complex and/or zygomatic arch fractures over a 71-month period. Dent Traumatol 2011;27:135-142.

5. Samad TA, Moore KA, Sapirstein A, Billet S, Allchorne A, Poole S, et al. Interleukin-1beta-mediated induction of Cox-2 in the CNS contributes to inflammatory pain hypersensitivity. Nature 2001;410:471-475.
6. Calderoni DR, Guidi Mde C, Kharmandayan P, Nunes PH. Seven-year institutional experience in the surgical treatment of orbito-zygomatic fractures. J Craniomaxillofac Surg 2011;39:593-599.

7. deSousa Santos JA, da Silva LC, de Santana Santos T, Menezes Junior LR, de Assuncao Oliveira AC, Brandao JR. Comparative study of tramadol combined with dexamethasone and diclofenac sodium in third-molar surgery. J Craniomaxillofac Surg 2012;40:694-700.

8. Aznar-Arasa L, Harutunian K, Figueiredo R, ValmasedaCastellon E, Gay-Escoda C. Effect of preoperative ibuprofen on pain and swelling after lower third molar removal: a randomized controlled trial. Int J Oral Maxillofac Surg 2012;41:1005-1009.

9. Ristow O, Pautke C, Kehl V, Koerdt S, Schwärzler K, Hahnefeld L. Influence of kinesiologic tape on postoperative swelling, pain and trismus after zygomatico-orbital fractures. Craniomaxillofac Surg 2013:1-8.

10. Assimes TL, Lessard ML. The use of perioperative corticosteroids in craniomaxillofacial surgery. Plastic and Reconstruct Surg 1999;103(1):313-321.

11. Ross R, White CP. Evaluation of hydrocortisone in prevention of postoperative complications after oral surgery: a preliminary report. J Oral Surg 1958;16(3):220-226.

12. Hussain A, Young WB. Steroids and aseptic osteonecrosis in migraine patients. Headache 2007;47(4):600-604.

13. Ularntinon S, Tzuang D, Dahl G, Shaw RJ. Concurrent treatmentof steroid-related mood and psychotic symptoms with risperidone. Pediatric 2010;125(5):e1241-1245.

14. Olsen M, Christensen S, RIIS A, Thomsen RW. Preadmission use of systemic glucocorticoids and 30-day mortality following bleeding peptic ulcer: a population-based cohort study. Am J Therapeutics 2010;17(1):23-29.

15. Williams S, Whatman C, Hume PA, Sheerin K. Kinesio taping in treatment and prevention of sports injuries: a meta-analysis of the evidence for its effectiveness. Sports Med 2012; 42:153-164.

16. Osunde OD, Adebola RA, Omeje UK. Management of inflammatory complications in third molar surgery: a review of the literature. Afr Health Sci 2011;11:530-537.

17. Rana M, Gellrich NC, von See C, Weiskopf C, Gerressen M, Ghassemi A, et al. Devaluation of postoperative swelling in treatment of bilateral mandibularfractures using 2 different cooling therapy methods: a randomized observer blind prospective study. J Craniomaxillofac Surg 2013;41(1):e17-23.

18. Thorén H, Snäll J, Kormi E, Numminen L, Fäh R, lizuka T, Lindqvist $\mathrm{C}$, Törnwall J. Does perioperative glucocorticosteroid treatment correlate with disturbance in surgical wound healing after treatment of facial fractures? A retrospective study. J Oral Maxillofac Surg 2009 Sep;67(9):1884-1888.

19. Derendorf H, Hochhaus G, Mollmann H, Barth J, Krieg M, Tunn S, Mollmann C. Receptor-based pharmacokineticpharmacodynamic analysis of corticosteroids. J Clin Pharmacol 1993;33(2):115-123.

20. Doksrod S, Sagen O, Nostdahl T, Raeder J. Dexamethasone does not reduce pain or analgesic consumption after thyroid surgery: a prospective, randomized trial. Acta Anaesthesiologica Scandinavica 2012;56(4):513-519.

21. WickeC,Halliday B, Allen D, Roche NS, ScheuenstuhlH, Spencer MM, Roberts AB, Hunt TK. Effects of steroids and retinoids on wound healing. Archives of Surg 2000;135(11):1265-1270.

22. Bronicki RA, Backer CL, Baden HP, Mavroudis C, Crawford SE, Green TP. Dexamethasone reduces the inflammatory response to cardiopulmonary bypass in children. The Annals of Thoracic Surg 2000;69(5):1490-1495. 